\title{
Long non-coding RNA JPX promotes gastric cancer progression by regulating CXCR6 and autophagy via inhibiting miR-197
}

\author{
XUEJING HAN ${ }^{1,2}$ and ZHENG LIU ${ }^{1}$ \\ ${ }^{1}$ Department of Digestion, The Second Affiliated Hospital of Nanjing Medical University, Nanjing, Jiangsu 210036; \\ ${ }^{2}$ Department of Digestion, The Affiliated Hospital of Xuzhou Medical University, Xuzhou, Jiangsu 221002, P.R. China
}

Received March 31, 2020; Accepted September 11, 2020

DOI: $10.3892 / \mathrm{mmr} .2020 .11698$

\begin{abstract}
Long non-coding RNAs (lncRNAs) serve a crucial role in gastric cancer (GC) progression. However, the molecular mechanism underlying lncRNA JPX transcript, XIST activator (JPX) in the tumorigenesis of GC is not completely understood. Reverse transcription-quantitative PCR (RT-qPCR) and western blotting were performed to detect gene expression. A luciferase reporter gene assay was conducted to determine the relationship between microRNA (miR)-197 and JPX or C-X-C motif chemokine receptor 6(CXCR6). Cell viability, migration and invasion were determined by performing MTT, wound healing and Transwell assays, respectively. The Cancer Genome Atlas database and the RT-qPCR results indicated that JPX expression was upregulated and miR-197 expression was downregulated in patients with GC and in GC cells. Moreover, high JPX expression and low miR-197 expression in patients with GC indicated poor prognosis. miR-197 expression was directly inhibited by JPX. Compared with the short hairpin RNA (sh) negative control (NC) group, NCI-N87 and MKN-45 cells in the shJPX group displayed decreased cell viability and invasion, as well as a wider scratch width. NCI-N87 and MKN-45 cells in the shJPX + miR-197 inhibitor group had increased viability and invasion, but a narrower scratch width compared with the shJPX group. It was also identified that miR-197 directly inhibited CXCR6 expression. miR-197 inhibited Beclin1 protein expression and promoted p62 protein expression. Compared with the NC group, NCI-N87 and MKN-45 cells in the miR-197 mimic group had decreased cell viability and invasion, and a wider scratch width. Enhanced cell viability and invasion, and a narrower scratch width was also observed in the miR-197 mimic + CXCR6 and miR-197 mimic + Beclin1 groups,
\end{abstract}

Correspondence to: Dr Zheng Liu, Department of Digestion, The Second Affiliated Hospital of Nanjing Medical University, 121 Jiangjiayuan Road, Xiaguan, Nanjing, Jiangsu 210036, P.R. China E-mail: zhengliu0808@163.com

Key words: long non-coding RNA JPX transcript, XIST activator, gastric cancer, microRNA-197, C-X-C motif chemokine receptor 6, autophagy compared with the miR-197 mimic group. Collectively, the results indicated that IncRNA JPX promoted GC progression by regulating CXCR6 and autophagy via inhibiting miR-197. Furthermore, JPX knockdown regulated GC cell phenotype by promoting miR-197.

\section{Introduction}

Gastric cancer (GC) is one of the most common malignancies and is the third leading cause of cancer-related mortality worldwide (1). In 2018, there were 1,033,701 new cases of GC diagnosed, which accounted for $5.7 \%$ of the total worldwide number of cancer diagnoses (2). Patients with GC often present with non-specific symptoms in the early stages and the majority of GC cases are diagnosed in the advanced stages, which generally indicates a poor prognosis (3). Clinically, despite advancements in the therapy of GC, the treatment effect remains unsatisfactory and the 5-year survival rate is $\leq 30 \%(4,5)$. The improvement in the prognosis of patients with GC requires the identification of novel and effective therapeutic targets. It has been reported that the pathogenesis of $\mathrm{GC}$ is caused by the altered expression of multiple genes (6). Therefore, the identification of tumor suppressor genes and oncogenes is of great importance for the diagnosis and treatment of GC.

Long non-coding RNAs (lncRNAs) are a class of RNA transcripts that are $>200$ nucleotides in length (7). lncRNAs lack protein-coding function, but exert physiological effects at the epigenetic, transcriptional and post-transcriptional levels (8). Previous studies have reported that a number of lncRNAs serve as crucial regulators in the progression of GC. For instance, Wu et al (9) revealed that lncRNA FEZF1 antisense RNA 1 (FEZF1-AS1) was highly expressed in GC tissues and cells, and was associated with poor prognosis in patients with GC. Therefore, lncRNA FEZF1-AS1 may serve as an independent prognostic factor in GC, which promotes the tumorigenesis of GC by activating the Wnt signaling pathway. By contrast, Nie et al (10) observed that lncRNA MIR31 host gene (MIR31HG) was downregulated in GC, which indicated poor prognosis in patients with GC. In vitro studies have also revealed that decreased lncRNA MIR31HG expression could promote GC cell proliferation (10). Similarly, GC associated transcript 2, transmembrane protein 238 like and maternally expressed 3 are considered as tumor suppressor genes in 
GC (11-13), whereas GC associated transcript 3, BC005927, urothelial cancer associated 1 and colon cancer associated transcript 1 were identified as cancer-promoting genes (14-17). Thus, the discovery of IncRNAs provides important therapeutic targets for GC.

IncRNA JPX transcript, XIST activator (JPX) is a member of IncRNA family that, to the best of our knowledge, has not been investigated in GC. In the present study, JPX expression was detected in GC and the effects of JPX on the development of GC were investigated. Several studies have revealed that microRNA (miRNA/miR)-197 acts as a tumor suppressor in the progression of GC. For example, Liao et al (18) reported that miR-197 suppressed GC progression by regulating metadherin, while Chen et al (19) indicated that hsa_circ_0092306 facilitated GG tumorigenesis by targeting miR-197. Moreover, increasing evidence has suggested that IncRNA could serve as a competing endogenous RNA against miRNA to regulate the progression of various types of cancer, such as cervical cancer, GC and ovarian cancer (20-22). However, whether JPX regulates miR-197 in GC requires further investigation. It has also been observed that $\mathrm{C}-\mathrm{X}-\mathrm{C}$ motif chemokine receptor 6 (CXCR6) is involved in the development of GC.

Therefore, the present study investigated whether JPX regulated the development of GC via the miR-197/CXCR6 axis, with the aim of identifying a novel potential target and theoretical basis for the treatment of GC.

\section{Materials and methods}

The Cancer Genome Atlas (TCGA) analysis. JPX and miR-197 expression levels in tumor and healthy tissues of patients with GC were downloaded from TCGA database (https://tcga-data. nci.nih.gov/tcga/). Differences in JPX and miR-197 expression levels between healthy and tumor tissues were analyzed.

Tissue specimens. A total of 32 paired GC and adjacent non-tumor tissues (3-5 $\mathrm{cm}$ adjacent to tumor) were collected from 32 patients with GC (mean age, 62 years; age range, 43-76 years; 17 male patients and 15 female patients) at The Second Affiliated Hospital of Nanjing Medical University between January 2016 and April 2018. The diagnoses of these samples were verified by experienced pathologists. Written informed consent was obtained from all patients. The present study was approved by the Ethics Committee of The Second Affiliated Hospital of Nanjing Medical University.

Cell culture. GC cell lines (NCI-N87 and MKN-45) and normal gastric mucosa epithelial cells (GES-1) were purchased from The Cell Bank of Type Culture Collection of the Chinese Academy of Sciences. Cells were cultured in $3 \mathrm{ml}$ DMEM (Gibco; Thermo Fisher Scientific, Inc.) supplemented with 10\% FBS (Gibco; Thermo Fisher Scientific, Inc.), $100 \mathrm{U} / \mathrm{ml}$ penicillin (Thermo Fisher Scientific, Inc.) and $100 \mu \mathrm{g} / \mathrm{ml} \mathrm{strep-}$ tomycin (Thermo Fisher Scientific, Inc.) in sterilized culture flasks at $37^{\circ} \mathrm{C}$ with $5 \% \mathrm{CO}_{2}$.

Transfection. The short hairpin RNA (shRNA) targeting JPX (shJPX; 5'-GUGCGUACAGUGCUGUACAGCAU-3') with its negative control (NC) shRNA (shNC; 5'-UACGCUCAG CAUGUGUCACUC-3'), miR-197 mimic (5'-UCGUUCGUG
AGCACUUGCGACG-3') with its NC mimics (5'-UCGUCG GAUCGACUGAGAUCU-3') and miR-197 inhibitor (5'-AGC CUUGCUGCAGGUGCGCAU-3') with its NC inhibitor (5'-UGCCUUACUGACGGUCGGAGA-3') were purchased from Shanghai GenePharma Co., Ltd. NCI-N87 and MKN-45 cells were transfected with $10 \mathrm{nM}$ shJPX and $10 \mathrm{nM}$ shNC. NCI-N87 and MKN-45 cells were transfected with $10 \mathrm{nM}$ miR-197 mimic and $10 \mathrm{nM} \mathrm{NC}$ to form the miR-197 mimic and $\mathrm{NC}$ groups, respectively.

CXCR6 and Beclin1 overexpression plasmids (pcDNA3.1-CXCR6 and pcDNA3.1-Beclin1, respectively) were established by inserting the full-length CXCR6 or Beclin1 sequence into the pcDNA3.1 vector (Thermo Fisher Scientific, Inc.). An empty pcDNA3.1 vector (pcDNA3.1) was used as the control.

NCI-N87 and MKN-45 cells were co-transfected with $10 \mathrm{nM}$ shJPX and $10 \mathrm{nM}$ miR-197 inhibitor (shJPX + miR-197 inhibitor group), miR-197 mimic and CXCR6 overexpression vector (miR-197 mimic + CXCR6 group) or miR-197 mimic and Beclin1 overexpression vector (miR-197 mimic + Beclin1 group).

All transfections were performed using Lipofectamine ${ }^{\circledR}$ 3000 reagent (Thermo Fisher Scientific, Inc.). Cells were maintained at $37^{\circ} \mathrm{C}$ and $5 \% \mathrm{CO}_{2}$ for $8 \mathrm{~h}$. Successfully transfected cells were screened and maintained in DMEM supplemented with $10 \% \mathrm{FBS}$ at $37^{\circ} \mathrm{C}$ and $5 \% \mathrm{CO}_{2}$. At $48 \mathrm{~h}$ post-transfection, cells were used for subsequent experimentation.

Luciferase reporter gene assay. StarBase 2.0 (http://starbase. sysu.edu.cn) was used to predict the potential miRNAs that can bind to JPX. Wild-type (WT) and mutant (MUT) JPX sequences, as well as WT and MUT CXCR6 sequences, were designed by Shanghai GenePharma Co., Ltd. and cloned into the psiCHECK2 luciferase reporter (Promega Corporation). NCI-N87 cells transfected with $10 \mathrm{nM}$ miR-197 mimics and $10 \mathrm{nM} \mathrm{NC}$ were prepared as single cell suspensions in serum-free DMEM at a density of $1 \times 10^{5}$ cells $/ \mathrm{ml}$. Cells were seeded in 6-well plates $(1 \mathrm{ml}$ cell suspensions per well). NCI-N87 cells were transfected with WT and MUT JPX psiCHECK2 luciferase reporters, as well as WT and MUT CXCR6 psiCHECK2 luciferase reporters. Transfection was performed using Lipofectamine 2000. At $48 \mathrm{~h}$ post-transfection, the luciferase activity of each well was determined using a Dual-luciferase reporter assay system (Promega Corporation) according to the manufacturer's protocol. The relative luciferase activity of each well was normalized to Renilla luciferase activity.

Reverse transcription-quantitative PCR (RT-qPCR). Tissue specimens were ground into powder in liquid nitrogen prior to RNA extraction. Total RNA was isolated from tissue specimens and cells using TRIzol ${ }^{\circledR}$ reagent (Thermo Fisher Scientific, Inc.). Total RNA (200 ng) was reverse transcribed into cDNA using SuperScript III (Invitrogen; Thermo Fisher Scientific, Inc.) at $37^{\circ} \mathrm{C}$ for $15 \mathrm{~min}$. Subsequently, qPCR was performed using an ABI PRISM 7500 Sequence Detection system (Thermo Fisher Scientific, Inc.) and SYBR Select Master mix (Thermo Fisher Scientific, Inc.). U6 or GAPDH were used as the internal reference genes. The following thermocycling conditions were used for qPCR: Initial denaturation at $95^{\circ} \mathrm{C}$ for $5 \mathrm{~min}$; followed by 40 cycles of $95^{\circ} \mathrm{C}$ for $20 \mathrm{sec}, 58^{\circ} \mathrm{C}$ for 
$30 \mathrm{sec}$ and $74^{\circ} \mathrm{C}$ for $30 \mathrm{sec}$ and a final extension step at $72^{\circ} \mathrm{C}$ for $5 \mathrm{~min}$. The primers used for qPCR were as follows: JPX forward, 5'-TGCAGTCAGAAGGGAGCAAT-3' and reverse, 5'-CACCGTCATCAGGCTGTCTT-3'; miR-197 forward, 5'-GTTCACCACCTTCTCCAC-3' and reverse, 5'-GTGCAG GGTCCGAGGT-3'; CXCR6 forward, 5'-ATGCCATGACCA GCTTTCACT-3' and reverse, 5'-TTAAGGCAGGCCCTC AGG-3'; Beclin1 forward, 5'-TAGGATCCATGGAAGGGT CTAAGAC-3' and reverse, 5'-GCGAAGCTTTCATTTGTT ATAAAAT-3'; GAPDH forward, 5'-GTCGATGGCTAGTCG TAGCATCGAT-3' and reverse, 5'-TGCTAGCTGGCATGC CCGATCGATC-3'; and U6 forward, 5'-GCTTCGGCAGCA CATATACTAAAA-3' and reverse, 5'-CGCTTCACGAAT TTGCGTGTCAT-3'. miRNA and mRNA expression levels were quantified using the $2^{-\Delta \Delta C q}$ method (23).

MTT assay. NCI-N87 and MKN-45 cells were re-suspended in DMEM supplemented with $10 \%$ FBS at a density of $1 \times 10^{5}$ cells $/ \mathrm{ml}$. Cell suspension $(100 \mu \mathrm{l})$ was added into 96-well plates for $0,24,48$ or $72 \mathrm{~h}$ at $37^{\circ} \mathrm{C}$ with $5 \% \mathrm{CO}_{2}$. At each time point, $20 \mu \mathrm{l}$ MTT solution $(5 \mathrm{mg} / \mathrm{ml}$; Sigma-Aldrich; Merck $\mathrm{KGaA}$ ) was added into each well for $4 \mathrm{~h}$ incubation at $37^{\circ} \mathrm{C}$. Subsequently, $150 \mu \mathrm{l} \mathrm{DMSO}$ was added to each well and the plated were gently agitated for $10 \mathrm{~min}$ at room temperature to dissolve the formazan crystal. The optical density of each well was measured at a wavelength of $490 \mathrm{~nm}$ using a microplate reader (Biotek Instruments, Inc.).

Wound healing assay. The wound healing assay was performed to determine cell migration. Briefly, NCI-N87 and MKN-45 cells re-suspended in DMEM supplemented with $1 \%$ FBS were inoculated into 6-well plates $(1 \mathrm{ml}$ cell suspension per well; $1 \times 10^{5}$ cells $\left./ \mathrm{ml}\right)$. Cells were incubated at $37^{\circ} \mathrm{C}$ with $5 \% \mathrm{CO}_{2}$ for $24 \mathrm{~h}$. Subsequently, a straight line was made through the center of each well using a $200 \mu \mathrm{l}$ pipette tip. The residual liquid in each well was discarded and replaced with fresh DMEM supplemented with 10\% FBS (24). Cells were incubated for $24 \mathrm{~h}$ at $37^{\circ} \mathrm{C}$ with $5 \% \mathrm{CO}_{2}$. The original wound width and wound width at $24 \mathrm{~h}$ was observed under a light microscope (magnification, x200) and analyzed using ImageJ software (version 1.48; National Institutes of Health).

Transwell experiment. NCI-N87 and MKN-45 cell invasion was assessed using Transwell chambers (pore size, $8 \mu \mathrm{m}$; EMD Millipore) precoated with $100 \mu \mathrm{l}$ Matrigel (BD Biosciences) for $1 \mathrm{~h}$ at room temperature. Cells $\left(5 \times 10^{4}\right)$ were plated in the upper chamber with $200 \mu 1$ serum-free DMEM. Into the lower chamber, $600 \mu \mathrm{l}$ DMEM containing $10 \%$ FBS was plated. The upper chambers were pre-coated with Matrigel for $1 \mathrm{~h}$ at room temperature. Following incubation for $24 \mathrm{~h}$ at $37^{\circ} \mathrm{C}$ with $5 \% \mathrm{CO}_{2}$, the Transwell chamber was removed and cells remaining on the upper chamber were gently removed using a cotton swab. Invading cells were fixed with $4 \%$ formaldehyde and stained with $0.1 \%$ crystal violet, both for $20 \mathrm{~min}$ at room temperature. Invading cells were observed using a light microscope (magnification, x200) in five non-overlapping random fields of view.

Western blotting. Total protein was extracted from NCI-N87 cells using RIPA cell lysis (Thermo Fisher Scientific,
Inc.) and quantified using a BCA kit (Thermo Fisher Scientific, Inc.). A total of $10 \mu \mathrm{g}$ proteins were separated via $10 \%$ SDS-PAGE and transferred to PVDF membranes, which were blocked with $5 \%$ skimmed milk for $2 \mathrm{~h}$ at room temperature. The membranes were incubated for $12 \mathrm{~h}$ at $4^{\circ} \mathrm{C}$ with primary antibodies targeted against: CXCR6 (1:1,000; cat. no. ab137134; Abcam), Beclin1 (1:1,000; cat. no. 3738; Cell Signaling Technology, Inc.), p62 (1:1,000; cat. no. 88588; Cell Signaling Technology, Inc.), LC3B (1:1,000; cat. no. ab51520; Abcam; containing LC3-I and LC3-II) and GAPDH (1:1,000; cat. no. ab8245; Abcam). The membranes were washed three times with TBS-0.1\% Tween-20 and subsequently incubated with horseradish peroxidase-conjugated secondary antibodies (1:1,000; goat anti-mouse IgG; cat. no. ab205719; and goat anti-rabbit IgG; cat. no. ab205718; Abcam) for $2 \mathrm{~h}$ at room temperature. Proteins were visualized using an enhanced chemiluminescence detection system (Amersham; Cytiva). Image-Pro ${ }^{\circledR}$ Plus software (version 6.0; Media Cybernetics, Inc.) was used to quantify the protein expression.

Statistical analysis. All experiments were repeated three times independently. Data are presented as the mean \pm SD. Statistical analyses were performed using SPSS software (version 19.0; IBM Corp). Comparisons between tumor and adjacent healthy tissue samples were performed using a paired Student's t-test, while comparisons between the experimental and control groups were conducted using an unpaired Student's t-test. Comparisons among multiple groups were analyzed using one-way ANOVA followed by Tukey's test. Kaplan-Meier survival curve analysis and the log-rank test were conducted to analyze the overall survival rate of patients. Patients were divided into high and low groups, as determined by the mean expression value. The correlation between JPX and miR-197 expression in patients with GC was analyzed using Pearson's correlation analysis. $\mathrm{P}<0.05$ was considered to indicate a statistically significant difference.

\section{Results}

High JPX expression and low miR-197 expression in patients with GC indicates poor prognosis. TCGA analysis demonstrated significantly upregulated JPX expression and downregulated miR-197 expression in patients with GC (Fig. 1A and B). Furthermore, JPX and miR-197 expression levels were measured in clinical GC tissues and adjacent non-tumor tissues. Consistently, the expression of JPX was significantly increased, whereas miR-197 was decreased in GC tissues (Fig. 1C and D). JPX expression was also higher and miR-197 expression was lower in GC cell lines (NCI-N87 and MKN-45) compared with a normal gastric mucosa epithelial cell line (GES-1; Fig. 1E and F).

The Pearson's correlation analysis indicated that JPX and miR-197 expression levels were moderately, negatively correlated in GC tissues (Fig. 1G). The Kaplan-Meier analysis suggested that high JPX expression or low miR-197 expression in patients were associated with a shorter overall survival time (Fig. 1H and I). Therefore, the results demonstrated that high JPX expression and low miR-197 expression in patients with GC indicated poor prognosis. 
A

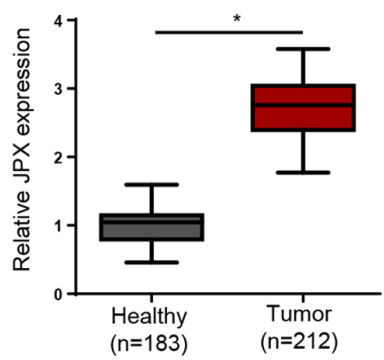

E

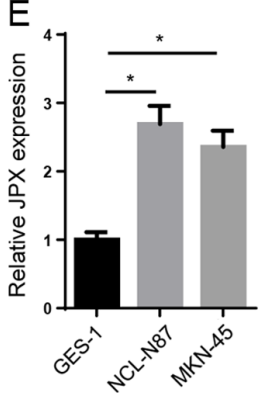

B

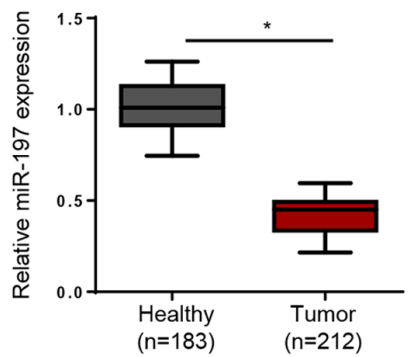

G

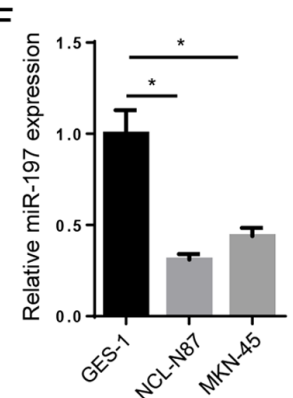

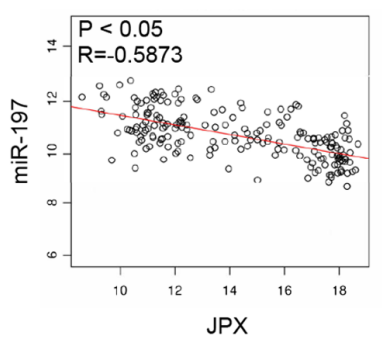

C

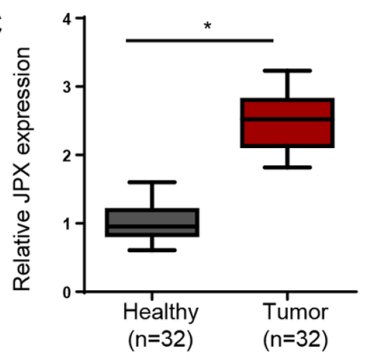

$\mathrm{H}$

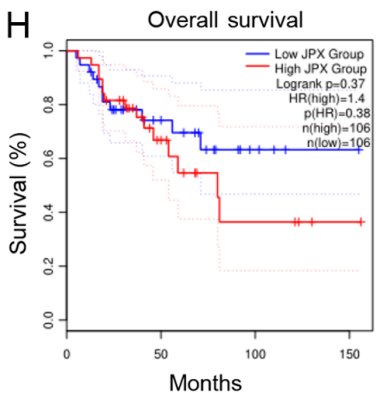

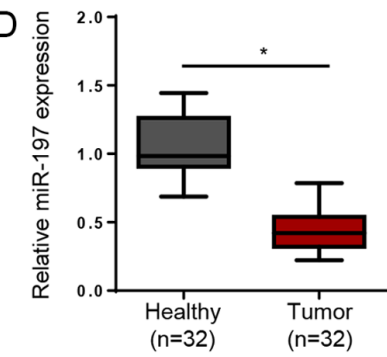

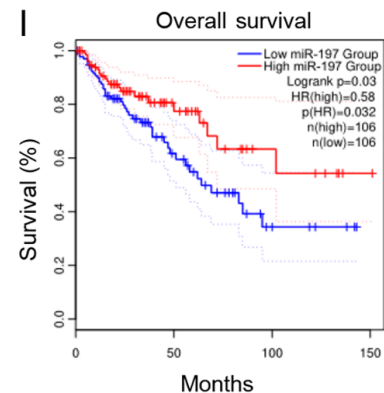

Figure 1. High JPX expression and low miR-197 expression in patients with GC indicates poor prognosis. (A) JPX and (B) miR-197 expression levels in patients with GC were determined using TCGA analysis. (C) JPX and (D) miR-197 expression levels in clinical GC tissues and adjacent non-tumor tissues $(n=32)$ were measured via RT-qPCR. (E) JPX and (F) miR-197 expression levels in GC cell lines (NCL-N87 and MKN-45) and normal gastric mucosa epithelial cell line (GES-1) were analyzed using RT-qPCR. (G) Based on data from TCGA, the correlation between JPX and miR-197 expression in patients with GC was analyzed using Pearson's correlation analysis. (H) Kaplan-Meier survival analysis revealed an association between JPX expression and overall survival rate of patients with GC. (I) Kaplan-Meier survival analysis revealed an association between miR-197 expression and overall survival rate of patients with GC. "P<0.05. GC, gastric cancer; TCGA, The Cancer Genome Atlas; miR, microRNA; JPX, long non-coding RNA JPX transcript, XIST activator; RT-qPCR, reverse transcription-quantitative PCR.

Knockdown of JPX inhibits GC cell activity, migration and invasion by promoting miR-197 expression. The StarBase results indicated that JPX may bind to miR-197 (Fig. 2A). The luciferase reporter gene assay further suggested that miR-197 overexpression significantly decreased the relative luciferase activity of WT JPX, but did not obviously alter the relative luciferase activity of MUT JPX (Fig. 2B). Moreover, miR-197 mimic significantly increased miR-197 expression, but markedly decreased JPX expression in NCI-N87 cells (Fig. 2C). RT-qPCR indicated that the expression levels of JPX or miR-197 were decreased in GC cells transfected with shJPX or miR-197 inhibitor, respectively (Fig. 2D and E). Compared with the shNC group, the shJPX group had higher miR-197 expression levels. However, compared with the shJPX group, the shJPX + miR-197 inhibitor group displayed significantly lower miR-197 expression (Fig. 2F). The results indicated that JPX bound to miR-197 and suppressed miR-197 expression.

The shJPX group demonstrated lower cell viability at $72 \mathrm{~h}$, a wider scratch width and decreased invasive abilities compared with the shNC group. However, compared with the shJPX group, the shJPX + miR-197 inhibitor group had a higher cell viability at $72 \mathrm{~h}$, a narrower scratch width and increased invasive abilities (Fig. 2G-I). Therefore, the results suggested that JPX knockdown inhibited GC cell activity, migration and invasion by promoting miR-197 expression.

miR-197 inhibits CXCR6 expression and autophagy. StarBase predicted that miR-197 possessed the binding site for CXCR6 (Fig. 3A). According to the luciferase reporter gene assay, miR-197 overexpression significantly inhibited the relative luciferase activity of WT CXCR6, but did not notably alter the relative luciferase activity of MUT CXCR6 (Fig. 3B). The miR-197 mimic group had higher miR-197 expression and lower CXCR6 expression (Fig. 3C). The western blotting results indicated that, compared with NC mimics, miR-197 overexpression decreased CXCR6 expression, the ratio of LC3-II/LC3-I and Beclin1 protein expression levels, but increased p62 protein expression in NCI-N87 cells (Fig. 3D and E).

miR-197 decreases GC cell activity, migration and invasion by inhibiting CXCR6 and Beclin1 expression levels. The expression levels of CXCR6 and Beclin1 were significantly upregulated in NCL-N87 cells transfected with pcDNA3.1/CXCR6 or pcDNA3.1/Beclin1 (Fig. 4A and B) Following miR-197 mimic transfection, NCI-N87 cells displayed markedly lower CXCR6 and Beclin1 expression levels. However, compared with the miR-197 mimic group, the miR-197 mimic + CXCR6 group displayed markedly higher CXCR6 expression and the miR-197 mimic + Beclin1 group displayed higher Beclin1 expression (Fig. 4C and D).

NCI-N87 and MKN-45 cell viability, migration and invasion were detected by performing CCK-8, wound healing and Transwell assays, respectively. The miR-197 mimic group displayed lower cell viability at $72 \mathrm{~h}$, a wider scratch width and declined invasive abilities compared with the NC group (Fig. 4E-G). However, compared with the miR-197 mimic group, the miR-197 mimic + CXCR6 and miR-197 mimic + Beclin1 groups had higher cell viability at $72 \mathrm{~h}$, a narrower scratch width and increased invasive abilities. Therefore, the results indicated that miR-197 inhibited GC cell activity, migration and invasion by inhibiting CXCR6 and Beclin1 expression levels. 
A

MUT JPX : 5' CAGUUAAUAGUAUUUCAACAC 3' WT JPX : 5' CAGUUAAUAGUAUUGUGGUGA $3{ }^{\prime}$ IIIII | hsa-miR-197 : 3' CAGUUAAUAGUAUUCACCACU 5'

\section{E}
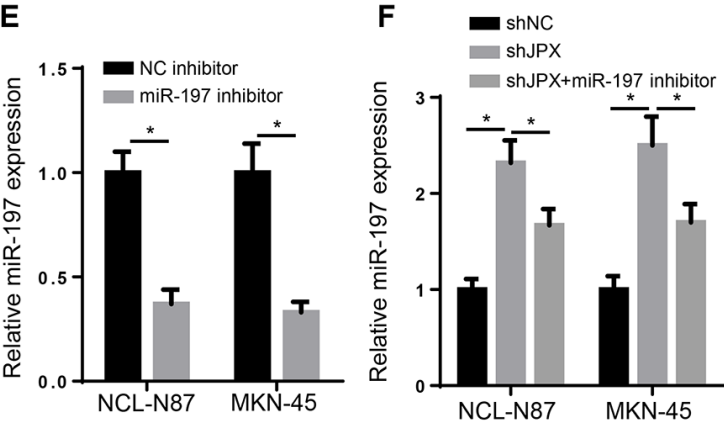

shJPX+

H
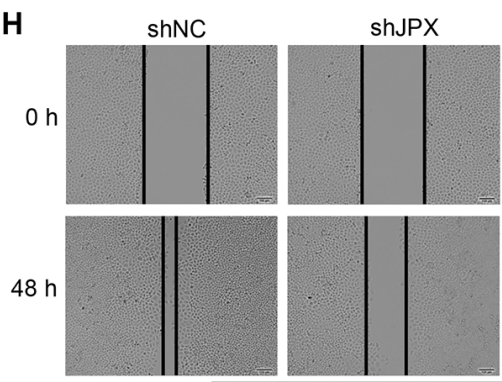

NCL-N87
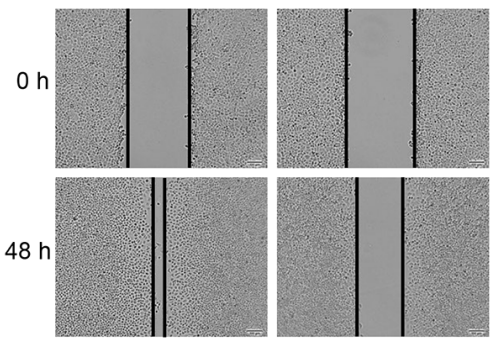

MKN-45

I
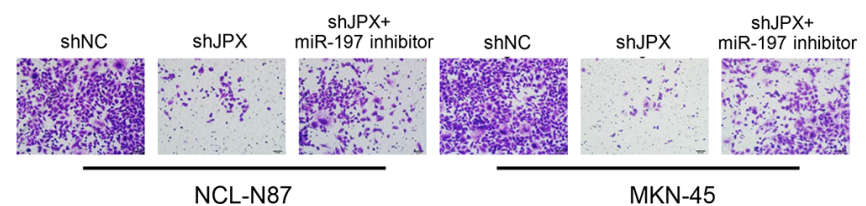
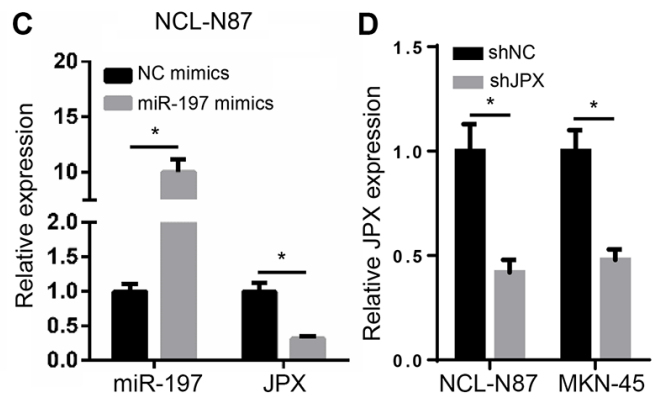

G

NCL-N87

MKN-45
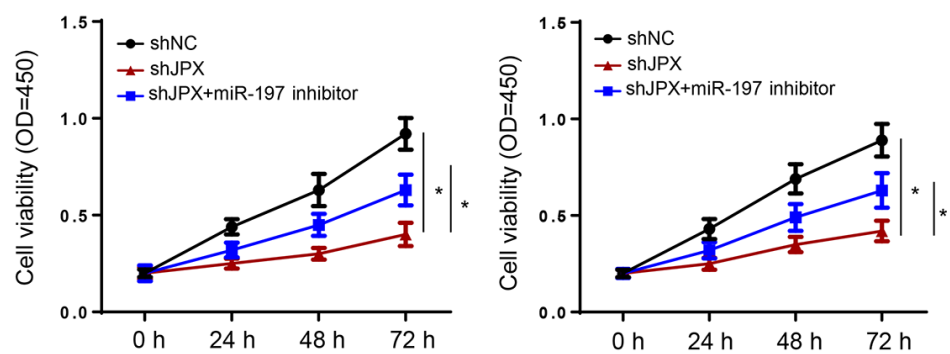

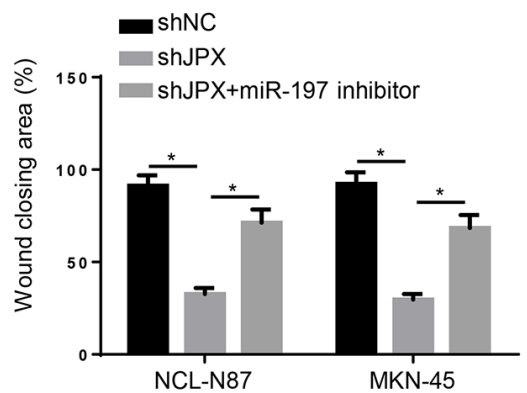

Figure 2. Knockdown of JPX inhibits the activity, migration and invasion of GC cells by promoting miR-197 expression. (A) Starbase predicted the targeted binding site between JPX and miR-197. (B) Luciferase reporter gene assay was used to confirm the relationship between JPX and miR-197. (C) RT-qPCR was performed to detect JPX and miR-197 expression levels in NCL-N87 cells. (D) RT-qPCR identified the expression of JPX in NCL-N87 and MKN-45 transfected with shNC and shJPX. (E) RT-qPCR demonstrated the expression of miR-197 in NCL-N87 and MKN-45 transfected with NC inhibitor and miR-197 inhibitor. (F) miR-197 expression in NCL-N87 and MKN-45 cells was assessed via RT-qPCR. (G) Viability of NCL-N87 and MKN-45 cells was determined using a Cell Counting Kit-8 assay. (H) Migration of cells was measured via a wound healing test (magnification, x200). (I) Invasion of cells was evaluated with Transwell assays (magnification, x200). ${ }^{*} \mathrm{P}<0.05$. miR, microRNA; JPX, long non-coding RNA JPX transcript, XIST activator; RT-qPCR, reverse transcription-quantitative PCR; NC, negative control; shRNA, short hairpin RNA; WT, wild-type; MUT, mutant; OD, optical density.

\section{Discussion}

The initiation and progression of human malignant tumors are associated with various factors, such as etiological factors, and genetic and epigenetic factors $(25,26)$. As important tumor regulators, IncRNAs can regulate the development of tumors at the epigenetic, transcriptional and post-transcriptional levels $(27,28)$. At present, the main mechanisms underlying 
A

MUT CXCR6: 5' UGUGCCCUCUUGAUUCAACAC 3' WT CXCR6: 5' UGUGCCCUCUUGAUGUGGUGA 3'

hsa-miR-197: 3' CGACCCACCUCUUCCACCACU 3,
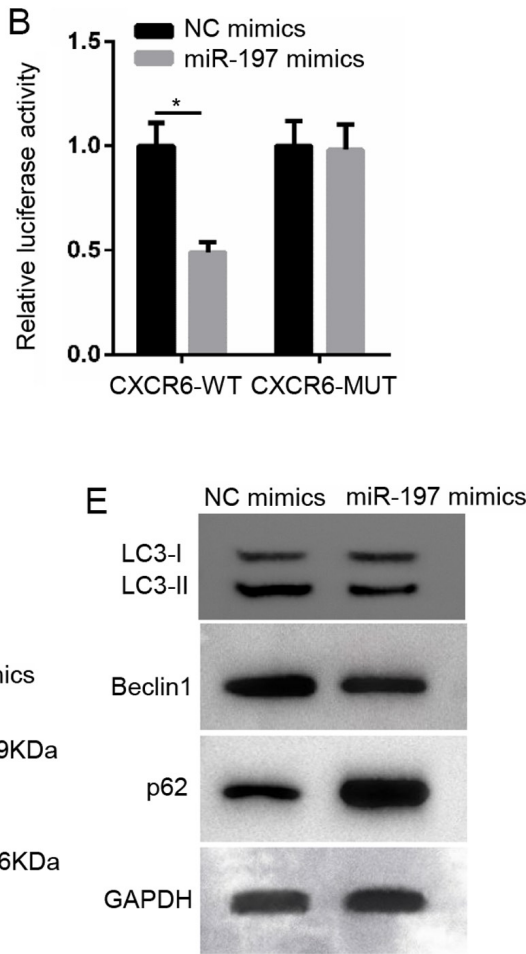

Figure 3. miR-197 directly inhibits CXCR6 expression and autophagy. (A) Starbase predicted the binding site between miR-197 and CXCR6. (B) Luciferase reporter gene assay was conducted to investigate the relationship between miR-197 and CXCR6. (C) miR-197 and CXCR6 expression levels in NCL-N87 cells were assessed via reverse transcription-quantitative PCR. (D) CXCR6, (E) LC3-II, LC3-I, Beclin1 and p62 protein expression levels were determined using western blotting. ${ }^{*} \mathrm{P}<0.05$. miR, microRNA; JPX, long non-coding RNA JPX transcript, XIST activator; NC, negative control; CXCR6, C-X-C motif chemokine receptor 6 .

lncRNAs affecting the development of GC are generally as follows: Some lncRNAs promote GC cell proliferation by inducing cell cycle arrest and inhibiting apoptosis, thereby affecting the progression of GC (29); moreover, several lncRNAs can inhibit or accelerate GC cell migration and invasion by affecting the epithelial-mesenchymal transition (30); lncRNAs also serve as endogenous competitive RNAs, which compete with miRNAs for binding to target genes, thereby regulating the downstream signaling pathways and the progression of GC (31); and finally, lncRNAs can promote the progression of GC via autophagy, metabolic stress and hypoxia (15). The present study suggested that JPX served as an endogenous competitive RNA, which promoted GC progression by regulating CXCR6 expression and promoting autophagy via sponging miR-197. To the best of our knowledge, the present study was the first to indicate that JPX served as a cancer-promoting gene in GC. Therefore, JPX may be a novel potential target for the treatment of GC.

The present study demonstrated that miR-197 was sponged and directly inhibited by JPX. Currently, the impact of miR-197 on the development of GC is not completely understood. Previous studies have investigated the effects of miR-197 on other human diseases. For instance, miR-197 serves as a cancer-promoting gene in bladder cancer, as indicated by the effect of miR-197 overexpression enhancing bladder cancer cell invasion and migration (32). Chen and Yang (33) revealed that miR-197-3p expression was significantly elevated in lung adenocarcinoma, and miR-197-3p inhibition suppressed lung adenocarcinoma cell proliferation and enhanced cell apoptosis. By contrast, TCGA analysis in the present study indicated that miR-197-3p expression in patients with GC was downregulated. Therefore, suggesting that miR-197-3p served as a tumor suppressor gene in GC, as evidenced by miR-197-3p overexpression attenuating cell proliferation, migration and invasion.

In the present study, the mechanism underlying miR-197-mediated inhibition of GC progression was suggested to occur via regulation of CXCR6 and autophagy. CXCR6 was identified to be a downstream gene of miR-197 and its expression was directly suppressed by miR-197. CXCR6 is a chemokine receptor of CXCL16 (34). CXCR6 is abnormally upregulated in multiple tumor tissues and cells, and the expression of CXCR6 is increased with the severity of tumor malignancy (35). In addition, CXCR6 has been reported to serve as a cancer-promoting gene in human tumors. For example, Ma et al (36) indicated that CXCR6 accelerated osteosarcoma cell proliferation and metastasis via the Akt signaling pathway. Gao et al (37) also demonstrated that CXCR6 upregulation contributed to a proinflammatory tumor microenvironment, which lead to metastasis and poor prognosis in hepatocellular carcinoma. Moreover, Jin et al (38) reported that CXCR6 expression in GC tissues was aberrantly increased, which was markedly associated with lymph node metastases and advanced clinical stage. In vitro studies have revealed that CXCR6 overexpression enhances GC cell migration and invasion (37). Li et al (39) also demonstrated that CXCR6 was significantly upregulated in patients with GC and GC cells. Moreover, the aforementioned study indicated that blocking CXCR6 signaling weakened GC cell migration and invasion. Consistent with the previous studies, the present 

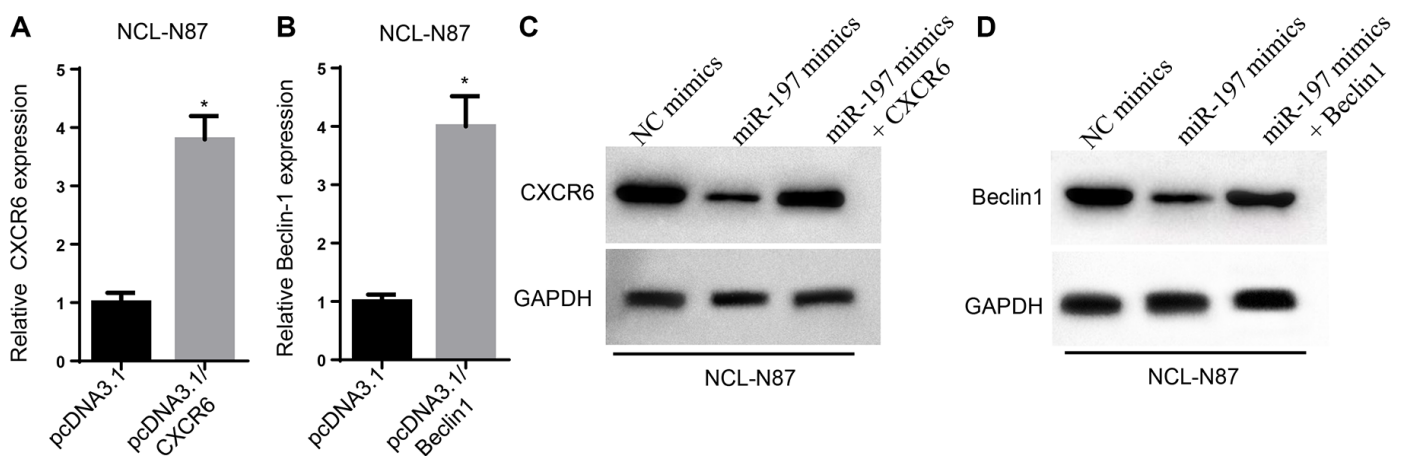

E
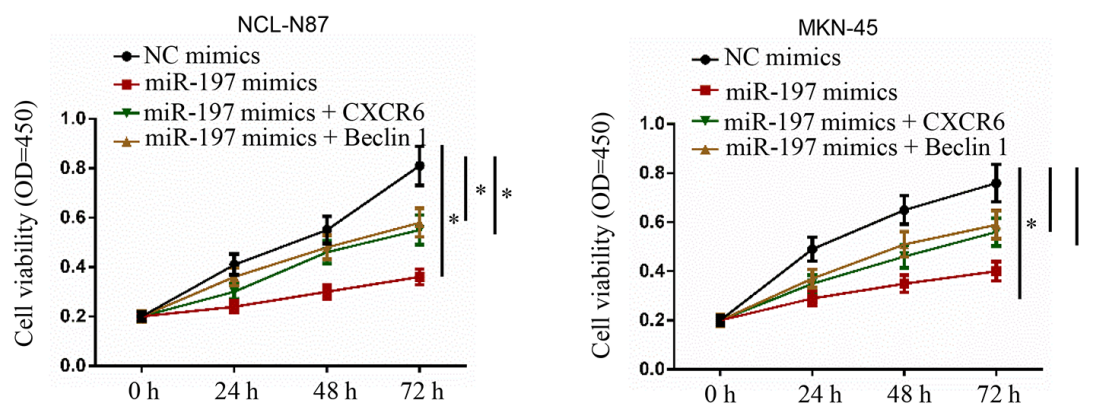

$\mathbf{F}$
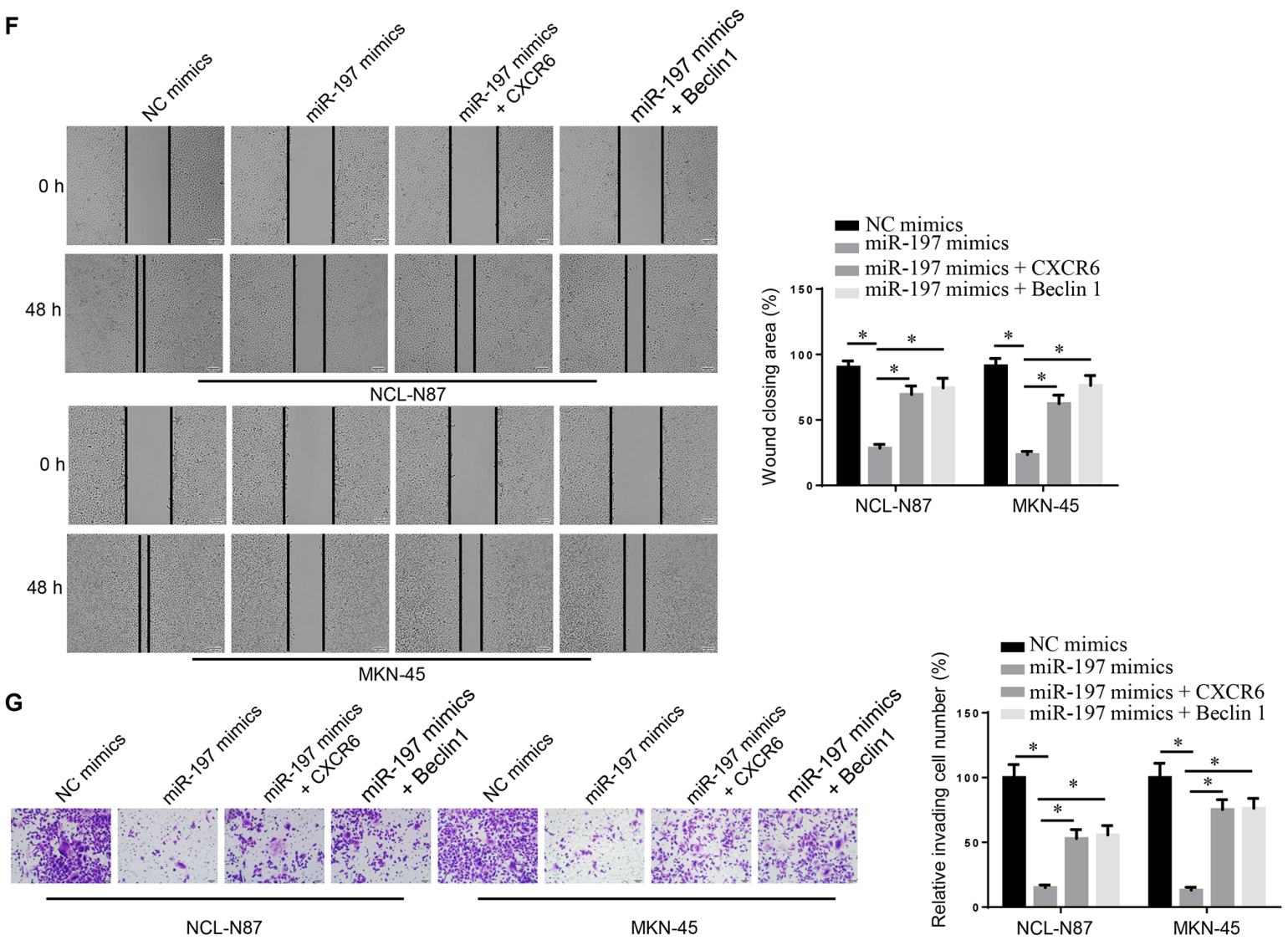

Figure 4. miR-197 inhibits the activity, migration and invasion of GC cells by decreasing CXCR6 and Beclin1 expression levels. (A) CXCR6 and (B) Beclin1 expression levels in NCL-N87 cells were assessed using reverse transcription-quantitative PCR. (C) CXCR6 and (D) Beclin1 expression levels were measured using western blot analysis. (E) Viability of NCL-N87 and MKN-45 cells was determined using a Cell Counting Kit-8 assay. (F) Migration of NCL-N87 and MKN-45 cells was measured via wound healing test (magnification, x200). (G) Invasion of NCL-N87 and MKN-45 cells was evaluated using Transwell assays (magnification, $\mathrm{x} 200$ ). ${ }^{*} \mathrm{P}<0.05$. miR, microRNA; JPX, long non-coding RNA JPX transcript, XIST activator; NC, negative control; CXCR6, C-X-C motif chemokine receptor 6; OD, optical density.

findings also suggested that CXCR6 was a cancer-promoting gene in GC. It was identified that CXCR6 overexpression in GC cells reversed the inhibitory effect of miR-197 on GC cell proliferation, migration and invasion.
Beclin1 and p62 are two important autophagy-related proteins $(40,41)$. Masuda et al (42) reported that autophagy was prominently associated with poor survival in patients with GC, especially for patients with stage I GC. Moreover, 
Geng et al (43) revealed that Beclin1 was upregulated in GC tissues and cells, and Beclin1 expression was an independent prognostic factor for patients with positive lymph node metastasis. Valencia et al (44) also observed that the expression of p62 was declined in the stroma of several tumors, and as an anti-inflammatory tumor suppressor, p62 could inhibit tumor development by modulating the metabolism in the tumor stroma. In addition, low p62 expression is closely associated with poorer outcomes in patients with colorectal cancer, as patients with low p62 expression display the most aggressive tumor features (45). Thus, p62 is considered as a candidate for autophagy-modulating therapies to treat tumors (46). In the present study, miR-197 overexpression decreased Beclin1 expression and increased p62 expression levels. Furthermore, Beclin1 overexpression reversed the inhibitory effect of miR-197 on GC cell viability, migration and invasion. Therefore, miR-197 may inhibit the development of GC by inhibiting autophagy.

However, there are limitations in the current study. Firstly, $10 \%$ FBS was used in the wound healing assay; however, cells should preferably be serum starved during wound healing assays. Secondly, all findings were based on in vitro assays; thus, in vivo experiments should be considered in future studies to elucidate the role of JPX in GC.

In conclusion, the present study investigated the effects of JPX in GC development and the results suggested that JPX was a cancer-promoting gene in GC. Regarding the underlying mechanism, JPX may promote the development of GC by regulating CXCR6 and enhance autophagy via inhibiting miR-197. Therefore, JPX may serve as a novel therapeutic target for GC.

\section{Acknowledgements}

Not applicable.

\section{Funding}

No funding was received.

\section{Availability of data and materials}

The datasets used and/or analyzed during the current study are available from the corresponding author on reasonable request.

\section{Authors' contributions}

$\mathrm{XH}$ and ZL designed the present study, performed the experiments, analyzed the data and prepared the figures, and drafted the initial manuscript. ZL reviewed and revised the manuscript. All authors read and approved the final manuscript.

\section{Ethics approval and consent to participate}

The present study was approved by the Ethics Committee of The Second Affiliated Hospital of Nanjing Medical University. Written informed consent was obtained from all patients.

\section{Patient consent for publication}

Not applicable.

\section{Competing interests}

The authors declare that they have no competing interests.

\section{References}

1. Li A, Li J, Lin J, Zhuo W and Si J: COL11A1 is overexpressed in gastric cancer tissues and regulates proliferation, migration and invasion of HGC-27 gastric cancer cells in vitro. Oncol Rep 37: 333-340, 2017.

2. Bray F, Ferlay J, Soerjomataram I, Siegel RL, Torre LA and Jemal A: Global cancer statistics 2018: GLOBOCAN estimates of incidence and mortality worldwide for 36 cancers in 185 countries. CA Cancer J Clin 68: 394-424, 2018.

3. Maimaiti Y, Maimaitiming M, Li Y, Aibibula S, Ainiwaer A, Aili A, Sun Z and Abudureyimu K: SSH1 expression is associated with gastric cancer progression and predicts a poor prognosis. BMC Gastroenterol 18: 12, 2018.

4. Wei GH and Wang X: lncRNA MEG3 inhibit proliferation and metastasis of gastric cancer via p53 signaling pathway. Eur Rev Med Pharmacol Sci 21: 3850-3856, 2017.

5. Dai F, Xuan Y, Jin JJ, Yu S, Long ZW, Cai H, Liu XW, Zhou Y, Wang YN, Chen Z and Huang H: CtBP2 overexpression promotes tumor cell proliferation and invasion in gastric cancer and is associated with poor prognosis. Oncotarget 8: 28736-28749, 2017.

6. Tsukamoto Y, Uchida T, Karnan S, Noguchi T, Nguyen LT, Tanigawa M, Takeuchi I, Matsuura K, Hijiya N and Nakada C: Genome-wide analysis of DNA copy number alterations and gene expression in gastric cancer. J Pathol 216: 471-482, 2008.

7. Ruan X, Li P and Cao H: Identification of transcriptional regulators that bind to long noncoding RNAs by RNA pull-down and RNA immunoprecipitation. Methods Mol Biol 1783: 185-191, 2018.

8. Liu K, Mao X, Chen Y, Li T and Ton H: Regulatory role of long non-coding RNAs during reproductive disease. Am J Transl Res 10: 1-12, 2018.

9. Wu X, Zhang P, Zhu H, Li S, Chen X and Shi L: Long noncoding RNA FEZF1-AS1 indicates a poor prognosis of gastric cancer and promotes tumorigenesis via activation of Wnt signaling pathway. Biomed Pharmacother 96: 1103-1108, 2017.

10. Nie FQ, Ma S, Xie M, Liu YW, De W and Liu XH: Decreased long noncoding RNA MIR31HG is correlated with poor prognosis and contributes to cell proliferation in gastric cancer. Tumour Biol 37: 7693-7701, 2016.

11. Shao Y, Chen H, Jiang X, Chen S, Li P, Ye M, Li Q, Sun W and Guo J: Low expression of lncRNA-HMlincRNA717 in human gastric cancer and its clinical significances. Tumour Biol 35: 9591-9595, 2014

12. Zeng S, Xie X, Xiao YF, Tang B, Hu CJ, Wang SM, Wu YY, Dong $\mathrm{H}$, Li BS and Yang SM: Long noncoding RNA LINC00675 enhances phosphorylation of vimentin on Ser83 to suppress gastric cancer progression. Cancer Lett 412: 179-187, 2018.

13. Sun M, Xia R, Jin F, Xu T, Liu Z, De W and Liu X: Downregulated long noncoding RNA MEG3 is associated with poor prognosis and promotes cell proliferation in gastric cancer. Tumour Biol 35: 1065-1073, 2014.

14. Shen W, Yuan Y, Zhao M, Li J, Xu J, Lou G, Zheng J, Bu S, Guo J and Xi Y: Novel long non-coding RNA GACAT3 promotes gastric cancer cell proliferation through the IL-6/STAT3 signaling pathway. Tumor Biol 37: 14895-14902, 2016.

15. Liu X, Wang Y, Sun L, Min J, Liu J, Chen D, Zhang H, Zhang H, Zhang H, Zhou Y and Liu L: Long noncoding RNA BC005927 upregulates EPHB4 and promotes gastric cancer metastasis under hypoxia. Cancer Sci 109: 988-1000, 2018.

16. Wang ZQ, He CY, Hu L, Shi HP, Li JF, Gu QL, Su LP, Liu BY, $\mathrm{Li} C$ and Zhu Z: Long noncoding RNA UCA1 promotes tumour metastasis by inducing GRK2 degradation in gastric cancer. Cancer Lett 408: 10-21, 2017.

17. Wang $P$, Liu X, Han G, Dai S, Ni Q, Xiao S and Huang J: Downregulated lncRNA UCA1 acts as ceRNA to adsorb microRNA-498 to repress proliferation, invasion and epithelial mesenchymal transition of esophageal cancer cells by decreasing ZEB2 expression. Cell Cycle 18: 2359-2376, 2019.

18. Liao Z, Li Y, Zhou Y, Huang Q and Dong J: MicroRNA-197 inhibits gastric cancer progression by directly targeting metadherin. Mol Med Rep 17: 602-611, 2018. 
19. Chen Z, Ju H, Zhao T, Yu S, Li P, Jia J, Li N, Jing X, Tan B and Li Y: hsa_circ_0092306 targeting miR-197-3p promotes gastric cancer development by regulating PRKCB in MKN-45 cells. Mol Ther Nucleic Acids 18: 617-626, 2019.

20. Luan $X$ and Wang Y: LncRNA XLOC_006390 facilitates cervical cancer tumorigenesis and metastasis as a ceRNA against miR-331-3p and miR-338-3p. J Gynecol Oncol 29: e95, 2018.

21. Zhang G, Li S, Lu J, Ge Y, Wang Q, Ma G, Zhao Q, Wu D, Gong W, Du M, et al: LncRNA MT1JP functions as a ceRNA in regulating FBXW7 through competitively binding to miR-92a-3p in gastric cancer. Mol Cancer 17: 87, 2018.

22. Liang H, Yu T, Han Y, Jiang H, Wang C, You T, Zhao X, Shan H, Yang R, Yang L, et al: LncRNA PTAR promotes EMT and invasion-metastasis in serous ovarian cancer by competitively binding miR-101-3p to regulate ZEB1 expression. Mol Cancer 17: 119, 2018.

23. Livak K and Schmittgen TD: Analysis of relative gene expression data using real-time quantitative PCR and the 2(-Delta Delta C(T)) method. Methods 25: 402-408, 2001

24. Yang Q, Yu Y, Sun Z and Pan Y: Long non-coding RNA PVT1 promotes cell proliferation and invasion through regulating miR-133a in ovarian cancer. Biomed Pharmacother 106: 61-67, 2018.

25. Giovannucci E: Modifiable risk factors for colon cancer. Gastroenterol Clin North Am 31: 925-943, 2002.

26. Cavatorta O, Scida S, Miraglia C, Barchi A, Nouvenne A, Leandro G, Meschi T, De' Angelis GL and Di Mario F: Epidemiology of gastric cancer and risk factors. Acta Biomed 89: 82-87, 2018.

27. Tan H, Zhang S, Zhang J, Zhu L, Chen Y, Yang H, Chen Y, An Y and Liu B: Long non-coding RNAs in gastric cancer: New emerging biological functions and therapeutic implications. Theranostics 10: 8880-8902, 2020.

28. Sun DE and Ye SY: Emerging roles of long noncoding RNA regulator of reprogramming in cancer treatment. Cancer Manag Res 12: 6103-6112, 2020

29. Pan L, Liang W, Gu J, Zang X, Huang Z, Shi H, Chen J, Fu M, Zhang P, Xiao X, et al: Long noncoding RNA DANCR is activated by SALL4 and promotes the proliferation and invasion of gastric cancer cells. Oncotarget 9: 1915-1930, 2017.

30. Liu YW, Sun M, Xia R, Zhang EB, Liu XH, Zhang ZH, Xu TP, De W, Liu BR and Wang ZX: LincHOTAIR epigenetically silences miR34a by binding to PRC2 to promote the epithelial-to-mesenchymal transition in human gastric cancer. Cell Death Dis 6: e1802, 2015

31. Mao Y, Liu R, Zhou H, Yin S, Zhao Q, Ding X and Wang H: Transcriptome analysis of miRNA-lncRNA-mRNA interactions in the malignant transformation process of gastric cancer initiation. Cancer Gene Ther 24: 267-275, 2017.

32. Wang YY, Wu ZY, Wang GC, Liu K, Niu XB, Gu S and Meng JS: LINC00312 inhibits the migration and invasion of bladder cancer cells by targeting miR-197-3p. Tumor Biol 37: 14553-14563, 2016.

33. Chen Y and Yang C: miR-197-3p-induced downregulation of lysine 63 deubiquitinase promotes cell proliferation and inhibits cell apoptosis in lung adenocarcinoma cell lines. Mol Med Rep 17: 3921-3927, 2018.

34. Darash-Yahana M, Gillespie JW, Hewitt SM, Chen YY, Maeda S, Stein I, Singh SP, Bedolla RB, Peled A, Troyer DA, et al: The chemokine CXCL16 and its receptor, CXCR6, as markers and promoters of inflammation-associated cancers. PLoS One 4: e6695, 2009
35. Deng L, Chen N, Li Y, Zheng H and Lei Q: CXCR6/CXCL16 functions as a regulator in metastasis and progression of cancer. Biochim Biophys Acta 1806: 42-49, 2010.

36. Ma Y, Xu X and Luo M: CXCR6 promotes tumor cell proliferation and metastasis in osteosarcoma through the Akt pathway. Cell Immunol 311: 80-85, 2017.

37. Gao Q, Zhao YJ, Wang XY, Qiu SJ, Shi YH, Sun J, Yi Y, Shi JY, Shi GM, Ding ZB, et al: CXCR6 upregulation contributes to a proinflammatory tumor microenvironment that drives metastasis and poor patient outcomes in hepatocellular carcinoma. Cancer Res 72: 3546-3556, 2012.

38. Jin JJ, Dai FX, Long ZW, Cai H, Liu XW, Zhou Y, Hong Q, Dong QZ, Wang YN and Huang H: CXCR6 predicts poor prognosis in gastric cancer and promotes tumor metastasis through epithelial-mesenchymal transition. Oncol Rep 37: 3279-3286, 2017.

39. Li Y, Fu LX, Zhu WL, Shi H, Chen LJ and Ye B: Blockade of CXCR6 reduces invasive potential of gastric cancer cells through inhibition of AKT signaling. Int J Immunopathol Pharmacol 28: 194-200, 2015

40. Zhai QL, Hu XD, Xiao J and Yu DQ: Astragalus polysaccharide may increase sensitivity of cervical cancer HeLa cells to cisplatin by regulating cell autophagy. Zhongguo Zhong Yao Za Zhi 43: 805-812, 2018 (In Chinese)

41. Xu Z, Yan Y, Zeng S, Qian L, Dai S, Xiao L, Wang L, Yang X, Xiao Y and Gong Z: Reducing autophagy and inducing G1 phase arrest by aloperine enhances radio-sensitivity in lung cancer cells. Oncol Rep 43: 1541-1548, 2017.

42. Masuda G, Yashiro M, Kitayama K, Miki Y, Kasashima H, Kinoshita H,Morisaki T, Fukuoka T, Hasegawa T, Sakurai K, et al: Clinicopathological correlations of autophagy-related proteins LC3, Beclin 1 and p62 in gastric cancer. Anticancer Res 36: 129-136, 2016.

43. Geng QR, Xu DZ, He LJ, Lu JB, Zhou ZW, Zhan YQ and Lu Y: Beclin-1 expression is a significant predictor of survival in patients with lymph node-positive gastric Cancer. PLoS One 7: e45968, 2012.

44. Valencia T, Kim JY, Abu-Baker S, Moscat-Pardos J, Ahn CS, Reina-Campos M, Duran A, Castilla EA, Metallo CM, Diaz-Meco MT and Moscat J: Metabolic reprogramming of stromal fibroblasts through $\mathrm{p} 62$-mTORC1 signaling promotes inflammation and tumorigenesis. Cancer Cell 26: 121-135, 2014.

45. Zhang J, Yang S, Xu B, Wang T, Zheng Y, Liu F, Ren F, Jiang J, Shi $\mathrm{H}$, Zou B, et al: p62 functions as an oncogene in colorectal cancer through inhibiting apoptosis and promoting cell proliferation by interacting with the vitamin D receptor. Cell Prolif 52: e12585, 2019

46. Adams O, Dislich B, Berezowska S, Schläfli AM, Seiler CA, Kröll D, Tschan MP and Langer R: Prognostic relevance of autophagy markers LC3B and p62 in esophageal adenocarcinomas. Oncotarget 7: 39241-39255, 2016.

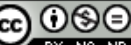

This work is licensed under a Creative Commons Attribution-NonCommercial-NoDerivatives 4.0 International (CC BY-NC-ND 4.0) License. 